\title{
A new shielding effectiveness measurement method based on a skin-effect transmission line coupler
}

\author{
T. Kleine-Ostmann, K. Münter, and T. Schrader \\ Physikalisch-Technische Bundesanstalt, Braunschweig, Germany
}

\begin{abstract}
We propose a new convenient material shielding effectiveness measurement method based on a skin-effect transmission line coupler. The method is somewhat similar to the arrangement with two coupled TEM cells known from literature. The transmission line coupler consists of a pair of identical transmission line 2-port devices. Each device contains a coaxial waveguide, with a circular inner conductor and an outer conductor having a square cross section. One side of the outer conductor is left completely open as a slot. The slot is surrounded by a large metal housing to contact the two halves. As a measure for the shielding effectiveness the coupling between the two devices is measured in terms of scattering parameters after the test material is brought between the two halves. The devices can be used in a range from low frequencies to a few $\mathrm{GHz}$.
\end{abstract}

\section{Introduction}

A variety of unconventional shielding materials finds vast applications in very diverse areas of our technical environment. Modern electronic equipment usually contains sensitive circuits and/or clock oscillators, and proper shielding of these components is often required to comply with electromagnetic compatibility regulations. Especially for high-volume production of consumer devices the conventional metal shielded enclosure is often prohibitively expensive, therefore alternatives had to be found, e.g. metal coated plastic materials. Another example of a rather unconventional shielding material is conductive textile used to make protective overalls for workers in possibly hazardous electromagnetic fields (Guy et al., 1987; Hoeft and Tokarsky, 2000). Also in traditional applications like shielded room construction new modular concepts rise the question how good the shielding effective-

Correspondence to: T. Kleine-Ostmann

(thomas.kleine-ostmann@ptb.de) ness of jointed metal panels (Fig. 1) is, which are screwed together. In any case, the shielding effectiveness of such materials can only be calculated for simple shielding geometries (Klinkenbusch, 2005). In most cases it must be measured, according to a suitable method.

Depending on the purpose, some methods based on cables (ASTM D4935, 1999; Kinningham and Yenni, 1988), coupled TEM cells (Nishikata et al., 2006) or antennas (IEEE Std 299, 1997; MIL Std 285, 1956; EN 50147-1, 1997; Wilson et al., 1986) are described in literature, all with specific advantages and disadvantages. In many cases the results depend on the individual test arrangement, and it is difficult to separate these influences and assign an uncertainty to the results. Obtaining material specific attenuation properties that are geometry independent remains difficult. The method and equipment described in the present paper is based on a transmission line coupler consisting of two separate halves between which a planar sample can be pressed (Fig. 2). It is similar to an arrangement with two coupled TEM cells, but easier to handle. However, it has also certain limitations. The measured material sample must be thin enough (thickness at least in the order of the skin depth) and must have sufficiently high conductivity from low frequencies to a few GHz. However, advantages are: calibration and traceability with a reference material, a small required sample size of only a few $\mathrm{cm}^{2}$, very little crosstalk and rapid measurements with a vector network analyzer (VNA), offering fast and simple traceable calibration.

In this paper we present our new measurement method and describe its numerical modelling based on the method of moments. In order to verify our calculations we compare shielding effectiveness measurements on steel foils of known thickness and conductivity to our numerical results. We find a good agreement of measurements and calculations, especially for low frequencies. Finally we present first applications of our new method by verifying the shielding effectiveness of assembled shielding panels and comparing the atten-

Published by Copernicus Publications on behalf of the URSI Landesausschuss in der Bundesrepublik Deutschland e.V. 


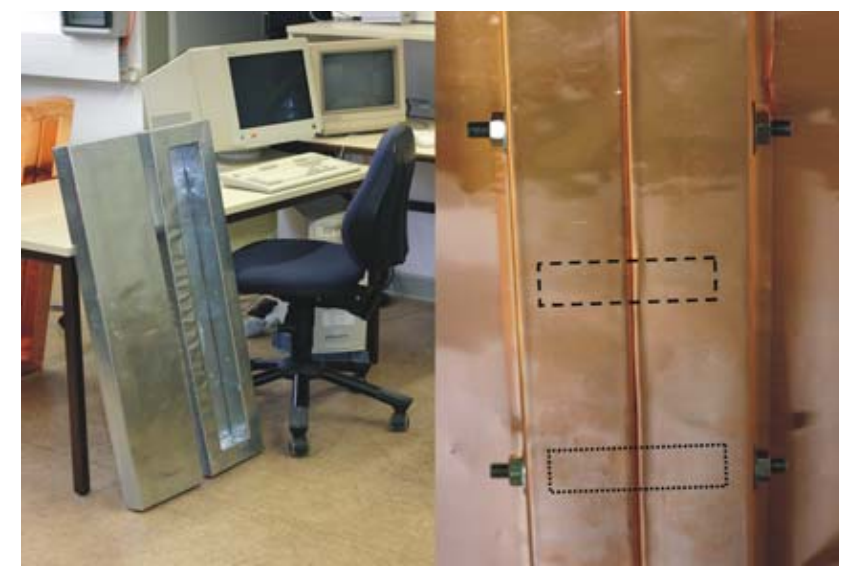

Fig. 1. Panels for modular construction of shielded rooms (left: separate zinc panels, right: copper panels screwed together). The measurement positions of the couplers at the position of a screw (dotted line) and between two screws (dashed line) are indicated.

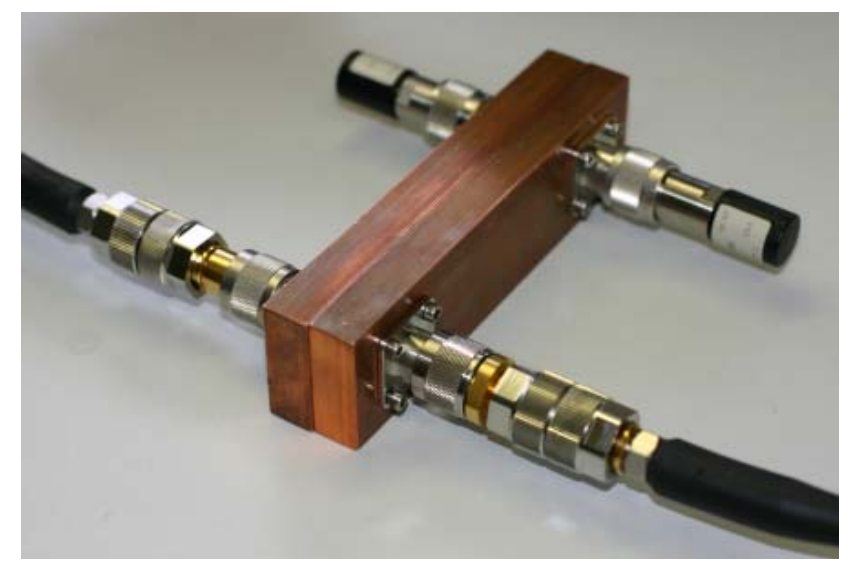

Fig. 2. Skin-effect transmission line coupler.

uation of different possible reflector materials for a new open area test site which is currently under construction.

\section{Measurement method and setup}

For the measurements a pair of identical transmission line 2-port devices are made from copper and fitted with $\mathrm{N}$ connectors (Fig. 2). Each device contains a coaxial waveguide, with a circular inner conductor (length $l=105 \mathrm{~mm}$, diameter $d=3 \mathrm{~mm}$ ) and an outer conductor having a square cross section $(6.5 \mathrm{~mm} \times 6.5 \mathrm{~mm}$, length $l=110 \mathrm{~mm})$. One side of the outer conductor is entirely left open as a slot, while the housing consists of a large metal area to contact the devices. If the slot is closed with a solid metal plate, the device is completed to become a precision $50 \Omega$ coaxial line with low insertion loss and a low input reflection coefficient. The

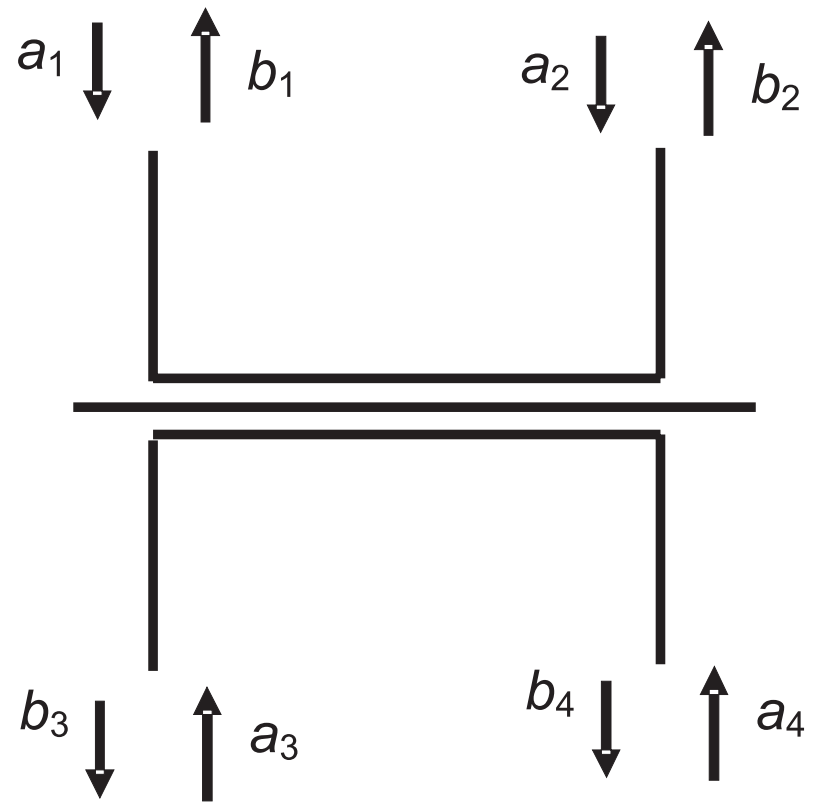

Fig. 3. Four-port representation of an ideal transmission line coupler.

measured input and output return loss shows that by the mechanical design, the coupler devices are well matched up to $3 \mathrm{GHz}$, ensuring a low voltage standing wave ratio (VSWR) on both coaxial lines. If both devices are attached to opposite sides of the separating metal plate, the crosstalk between the two coaxial compartments of the 4-port is strongly reduced depending on the shielding properties of the material. If the metal plate is removed and the devices are directly connected, there is a maximum coupling between the electric and magnetic fields in the two slots. In this configuration the 4-port behaves like a conventional directional coupler - but, of course, here directivity and bandwidth are not optimized for that purpose. Fig. 3 shows the 4-port representation of the transmission line coupler indicating the waves $a_{1}, \ldots, a_{4}$ in the forward direction and $b_{1}, \ldots, b_{4}$ in the backward direction. In the ideal case it is loss-less and reciprocal. Therefore it can be described by scattering parameters $S_{i k}=S_{k i}$ with

$$
\left(\begin{array}{l}
b_{1} \\
b_{2} \\
b_{3} \\
b_{4}
\end{array}\right)=\left(\begin{array}{ccccc}
S_{11} \approx 0 & S_{12} \approx 1 & S_{13} & S_{14} \\
S_{21} \approx 1 & S_{22} \approx 0 & S_{23} & S_{24} \\
S_{31} & S_{32} & S_{33} \approx 0 & S_{34} \approx 1 \\
S_{41} & S_{42} & S_{43} \approx 1 & S_{44} \approx 0
\end{array}\right) \cdot\left(\begin{array}{l}
a_{1} \\
a_{2} \\
a_{3} \\
a_{4}
\end{array}\right)
$$

Of especial importance are the coupling strengths $S_{31}$ and $S_{41}$ since they can be used to characterize the shielding effectiveness of the tested material. In the case of directly connected couplers without metal plate between them, $\left|S_{41}\right|$ is in the range of $-50 \mathrm{~dB}$ whereas $\left|S_{31}\right|$ varies between $-25 \mathrm{~dB}$ and $-50 \mathrm{~dB}$, showing oscillatory behavior as a result of the resonance length of the device. 


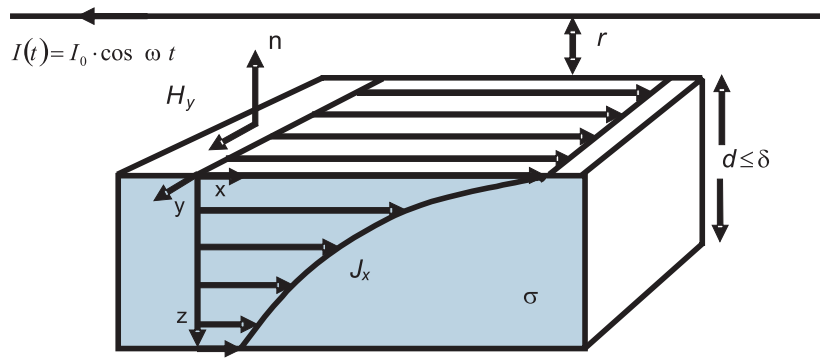

Fig. 4. Energy transport through metallic shielding material (surface normal $\mathrm{n}$ and distance $r$ to the circular conductor) with specific conductivity and with thickness $d$ smaller than the skin-depth $\delta$. The magnetic field $H_{y}$ of the circular conductor with alternating current $I(t)$ of amplitude $I_{0}$ and angular frequency $\omega$ causes a current density $J_{x}$ in the shielding material.

Shielding effectiveness measurements can only be made in comparison to a reference material. The first reason why it is not desirable to use the direct connection as $0 \mathrm{~dB}$-reference for measurements of shielding material is that the devices are no longer $50 \Omega$ lines. The second reason follows from the fact that the coupling mechanism is different, if very thin, but highly conductive material is inserted between the two devices. As with the bulk metal plate described above, both sides are then again $50 \Omega$ lines. The excitation signal on one side causes a current distribution which is nearly the same as if the coaxial lines were completely separated. In this case the coupling into the second compartment is no longer caused by electromagnetic fields in air, instead it may be best described by the skin effect, which makes a small fraction of the current distribution on the excited input line side "visible" at the other surface of the thin material. This situation is depicted in Fig. 4. The propagation of external fields into a conductive material under these conditions is discussed by Jackson (2002). The current density in the conductive material decays with the distance from the surface where the excitation takes place. The coaxial line at the output side is excited by a low-impedance, travelling wave current distribution on one of its surfaces - a situation which is difficult to model with conventional transmission line theory, because distributed generators would be required.

To test these assumptions, measurements as well as calculations based on a numerical simulation model were performed for the coupling devices described above, separated by foils of different thickness made from stainless steel. Materials of $10 \mu \mathrm{m}, 20 \mu \mathrm{m}, 25 \mu \mathrm{m}, 30 \mu \mathrm{m}$ and $40 \mu \mathrm{m}$ thickness were available for this purpose.

The two ports of the VNA were connected to one port of each device, with the other port of the transmission lines each terminated with a precision $50 \Omega$ load resistor. Two different arrangements were then measured, one with the port connectors in close proximity (referred as the forward direction, ports 1 and 3 as in Fig. 3), and the other with one line in

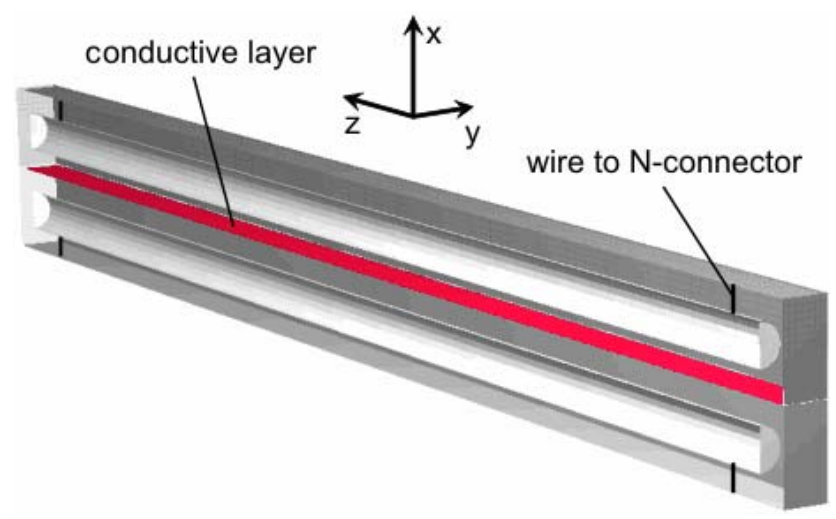

Fig. 5. Computer model of the skin-effect transmission line coupler.

reverse direction (ports 1 and 4 as in Fig. 3), with the VNA ports at opposite ends.

\section{Numerical simulation model}

For the numerical evaluation of the transmission properties of the empty coupler and the coupler with foil the method of moments implemented in the program Concept II (Singer and Brüns, 2006) seemed appropriate. This numerical program code offers a special feature, which seemed best suited to calculate the transmission through highly reflective and very thin layers. It accurately calculates the penetration of waves through highly reflective materials with high shielding attenuation - provided that the material is thin and highly conductive.

Under these circumstances the energy flow inside the material is perpendicular to the surface, and its propagation is well described by a transmission-line approach, where the attenuation along the line is derived from the skin-effect formula.

The problem was to define a geometrical model which was accurate enough to ensure an unidirectional energy flow and a proper smooth current distribution along the excited transmission line. Figure 5 shows the simulated geometry. The model consists of two different dielectric bodies of $r=1$ with ideally conducting metallic surfaces and embedded conductors which represent the two coupler halves. The connections from the $\mathrm{N}$-connector to the inner conductor are simply modelled by $1 \mathrm{~mm}$ thick wires of $5 \mathrm{~mm}$ length which are connecting the conductors to the outer surface of the slot waveguide in which they are embedded. All wires are loaded with a $50 \Omega$ impedance. A signal of constant power is fed in port 1. Scattering parameters are derived from the currents observed on the wires.

The two halves are separated by a conductive layer that represents the sample under investigation for which thickness and conductivity can be specified. The implementation 


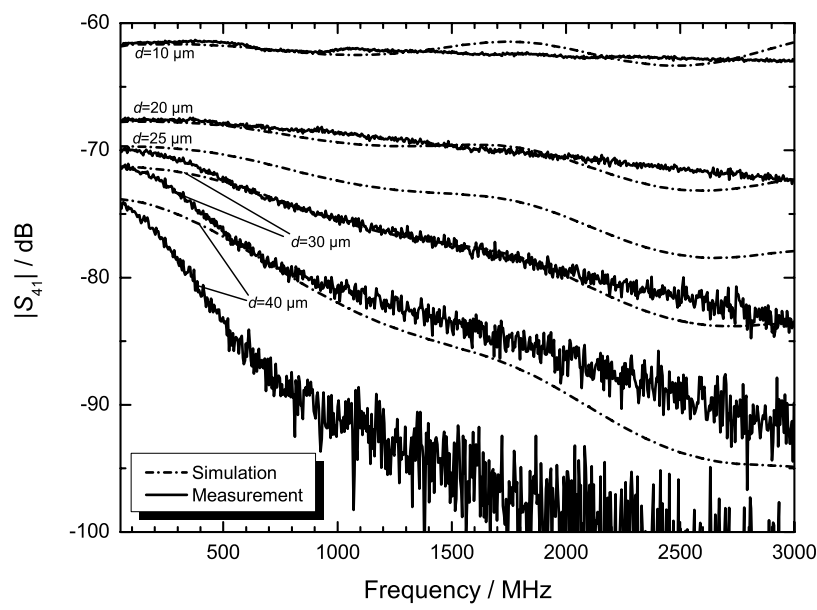

Fig. 6. Comparison between shielding measurements and numerical simulations for $S_{41}$.

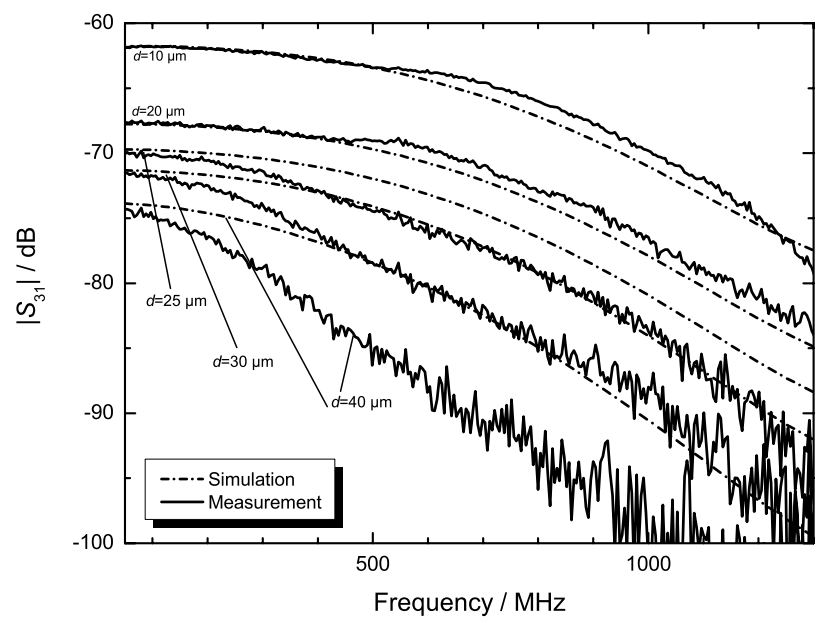

Fig. 7. Comparison between shielding measurements and numerical simulations for $S_{31}$.

benefits from the symmetry of the devices with regard to the cross section shown in Fig. 5 which reduces the amount of variables and computation time by a factor of two. The simulations were performed with approximately $13500 \mathrm{com}$ plex variables on a PC with 2 GByte RAM using swap files on the hard disk. A single simulation run involved 60 frequency steps of $50 \mathrm{MHz}$ up to $3 \mathrm{GHz}$ and took approximately three days.

\section{Comparison of measurements and simulations}

We performed measurements with stainless steel foils of known thickness and conductivity to be able to relate material properties to their measured coupling strength and to

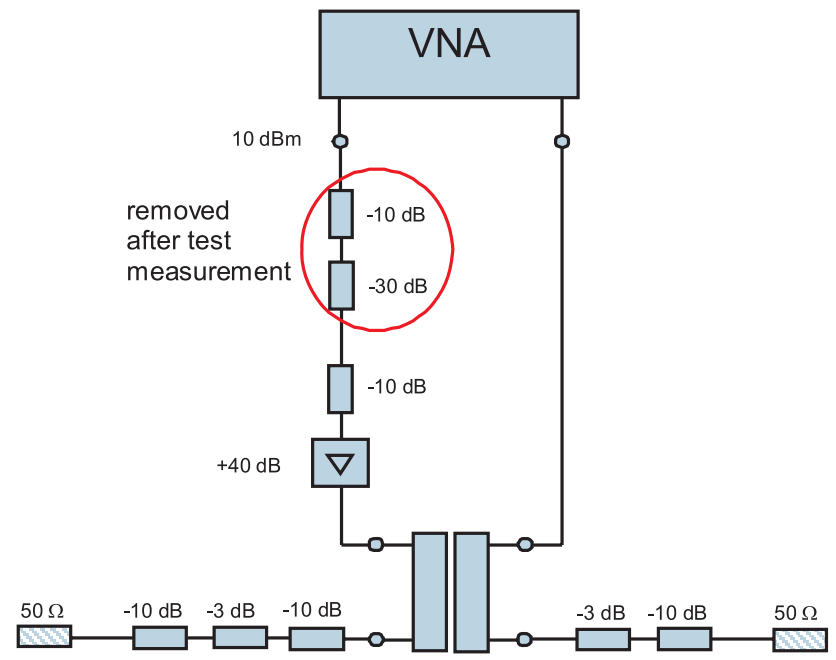

Fig. 8. Measurement setup with increased dynamics.

verify our numerical simulations. The measurement dynamics are in the order of $90 \mathrm{~dB}$. Figure 6 shows a comparison of measurements and simulations for the transmission in reverse direction $\left(S_{41}\right)$ whereas Fig. 7 shows the corresponding comparison for the forward direction $\left(S_{31}\right)$. Before the measurement the VNA was calibrated. The $0 \mathrm{~dB}$-line corresponds to a through connection between the VNA ports.

In both cases we see a good agreement between measurements and simulations for the lower frequencies, especially for $10 \mu \mathrm{m}$ and $20 \mu \mathrm{m}$ thick foils. As the frequency increases, the discrepancy between simulations and measurements becomes more pronounced, especially in the case of $S_{31}$. We attribute this to the fact that the modelled geometry lacks a sufficient level of detail to represent standing waves in the output section correctly. Furthermore, the measured coupling ranges below the simulated results. Obviously losses are not sufficiently accounted for as the coupler housing is assumed to be ideally conducting. Therefore, the reverse $\left(S_{41}\right)$ direction should be preferred for the measurement of the shielding effectiveness. For thin foils $(10 \mu \mathrm{m}$ and $20 \mu \mathrm{m}$ thickness), the coupling can be calculated with good agreement between theory and experiment up to $3 \mathrm{GHz}$, whereas for thicker foils accurate calculations are restricted to a few hundred $\mathrm{MHz}$, depending on the thickness. The results show that the measured coupling strength can be reliably related to the thickness of the foil.

\section{Applications}

First, we use our new method to verify the shielding effectiveness of the panels shown in Fig. 1. The panels under investigation were stringed either with copper or with zinc foil. While the $200 \mu \mathrm{m}$ thick foil has almost perfect shielding properties in both cases (beyond the limits set by the mea- 


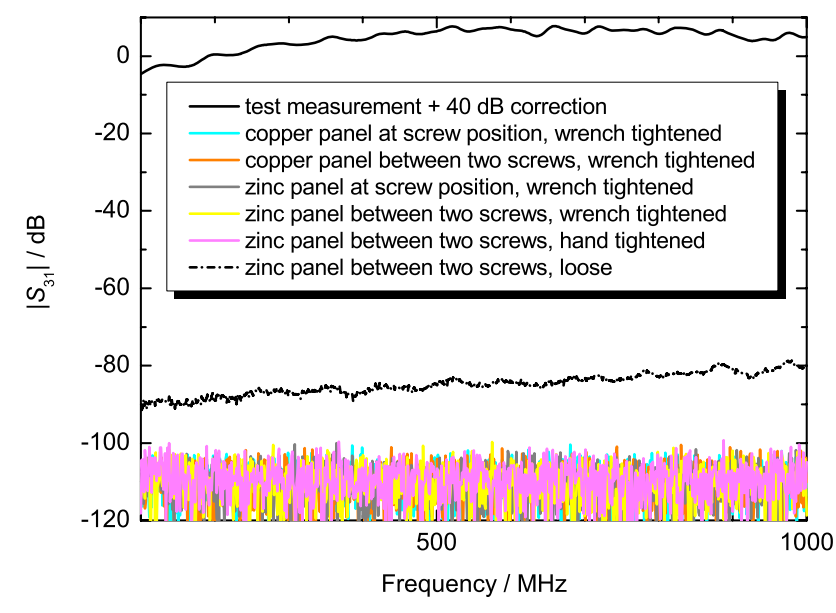

Fig. 9. Attenuation of different panel configurations.

surement dynamics), the connection between panels seems critical. The interface between two adjacent panels is simply established by screwing panels together. This way blank copper or zinc surfaces on the panel sides are pressed together.

In order to achieve higher measurement dynamics compared to a simple VNA measurement, we use the setup shown in Fig. 8. Precision attenuators of $50 \mathrm{~dB}$ followed by a $40 \mathrm{~dB}$ amplifier (Amplifier Research 10W1000) are connected to port 1 of the VNA. After calibration of the VNA and a test measurement of the empty coupler two of the attenuators are removed, increasing the measurement dynamics by 30 $\mathrm{dB}$. Next, the couplers were placed orthogonally across the interconnection of two panels (see Fig. 1), forcing the currents to flow across the contact area. Figure 9 shows the results for different measurement positions (at the position of a screw and between two screws) and for different attachment strengths (loose, hand tightened and wrench tightened), both for copper and zinc panels. Only in the case of a loose screw which led to a visible crack between panels, a decoupling of approximately $80 \mathrm{~dB}$ was measured. In all other cases the decoupling was beyond the measurement dynamics of at least $100 \mathrm{~dB}$.

As a second application we compare the shielding effectiveness of different surface coating materials. This measurement was done to evaluate the performance of possible reflector materials for a new open area test site which is currently under construction at the Physikalisch-Technische Bundesanstalt. Since we were not sure that a thin metal coating is sufficiently reflecting we tested its transparency for electromagnetic waves assuming that an intransparent metal layer would reflect most of the incoming radiation. Figure 10 shows the coupling factor $S_{41}$ for metallized steel cloth, a double layer of anti-static foil, and two different zinc metal layers sprayed on paper using a electro-thermal process compared to the previously measured $10 \mu \mathrm{m}$ zinc foil. The $50 \mu \mathrm{m}$

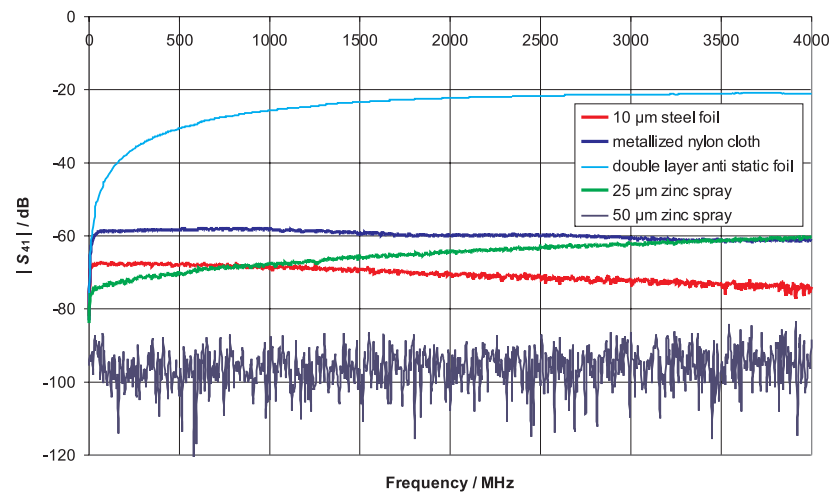

Fig. 10. Attenuation of different possible reflector materials for open area test sites.

zinc layer causes a complete decoupling, already. Although our method does not measure its reflectivity, good reflection properties of such a layer can be assumed as the transmission parameter $\left|S_{21}\right|$ is close to $0 \mathrm{~dB}$, representing a low attenuation of the signal along the line. A comparable zinc layer will be used for the new open area test site in Braunschweig.

\section{Conclusions}

We demonstrated a new method to evaluate the shielding effectiveness of planar materials, based on a transmission line coupler. Couplers are broadband devices with well-matched transmission lines at both VNA ports, reducing uncertainty in measured transmission S-parameters. Compared to other methods, it is much easier to handle since it avoids antennas or resonant cavities and allows fast network analyzer measurements. Furthermore, with other methods the reflection coefficients at the VNA ports change significantly, when the material sample is inserted. The measurements can be used to specify a reference material to which other shielding materials can be compared.

Measured and calculated transmission coefficients for the transmission line couplers and different metallic shielding materials are in reasonable agreement already (especially at the low-frequency end), which indicates that the numerical model is adequate and suited for the problem discussed here. However, a further refinement of the simulated geometry will help to achieve a better match between measurements and theory. Further work that aims to relate material properties directly to the observed coupling is under way.

Acknowledgements. The authors thank H. Brüns from the Institute of Electromagnetic Theory at Technical University HamburgHarburg for his advice regarding the electromagnetic simulation of the device and A. Daneschnejad as well as Thomas Baron from the Physikalisch-Technische Bundesanstalt for the precise fabrication of the transmission line coupler. 


\section{References}

ASTM D4935: Standard Test Method for Measuring the Electromagnetic Shielding Effectiveness of Planar Materials, 1999.

EN 50147-1: Anechoic chambers, Shield attenuation measurement, 1997.

Guy, A. W., Chung-Kwang Chou, McDougall, J. A., and Sorensen, C.: Measurement of Shielding Effectiveness of MicrowaveProtective Suits, IEEE Trans. Microwave Theory Techn., 35, 984-994, 1987.

Hoeft, L. O. and Tokarsky, E. W.: Measured Electromagnetic Shielding Characteristics of Fabric Made from Metal Clad Aramid Yarn and Wire, EMC 2000 Symp. Rec., 2, 883-886, 2000.

IEEE Std 299: IEEE Standard Method for Measuring the Effectiveness of Electromagnetic Shielding Enclosures, 1997.

Jackson, J. D.: Klassische Elektrodynamik, De Gruyter, Berlin, 2002.
Kinningham, B. A. and Yenni, D. M.: Test methods for electromagnetic shielding materials, IEEE Intern. Symp. Electromagn. Comp., 223-230, 1988.

Klinkenbusch, L.: On the Shielding Effectiveness of Enclosures, IEEE Trans. Electromagn. Comp., 47, 589-601, 2005.

MIL Std 285: Attenuation Measurements for Enclosures, Electromagnetic Shielding, for Electronic Test Purposes, Method of, 1956.

Nishikata, A., Saito, R., and Yamanaka, Y.: Low Frequency Equivalent Circuit of Dual TEM Cell for Shielding Material Measurement, IEICE Trans. Electron., E89-C, 44-50, 2006.

Singer, H. and Brüns, H.: Concept II, www.tet.tu-harburg.de, 2006.

Wilson, P. F., Adams, J. W., and Ma, M. T.: Measurement of the electromagnetic shielding capabilities of materials, Proc. IEEE, 74, 112-115, 1986. 